\title{
A COMPARISON OF TREE SEGMENTATION METHODS USING VERY HIGH DENSITY AIRBORNE LASER SCANNER DATA
}

\author{
F. Pirotti ${ }^{\mathrm{a}, \mathrm{b}}$, M. Kobal $^{\mathrm{c}}$, J.R. Roussel $^{\mathrm{d}}$ \\ a CIRGEO, Interdepartmental Research Center of Geomatics, University of Padua, Viale dell'Università 16, 35020 Legnaro, Italy \\ b TESAF Department, University of Padua, Viale dell'Università 16, 35020 Legnaro, Italy - francesco.pirotti@ unipd.it \\ ${ }^{\mathrm{c}}$ Department of Forestry and Renewable Forest Resources, Biotechnical Faculty, University of Ljubljana, Večna pot 83, 1000 \\ Ljubljana, Slovenia - milan.kobal@bf.uni-lj.si \\ ${ }^{\mathrm{d}}$ Centre de recherche sur les matériaux renouvelables, Département des sciences du bois et de la forêt, Pavillon Gene-H.-Kruger, \\ 2425 rue de la Terrasse, Université Laval, Québec, QC G1V 0A6, Canada - jean-romain.roussel.1@ulaval.ca
}

Commission III, WG III/10

KEY WORDS: Airborne laser scanner, LiDAR, tree segmentation, dense point cloud, forestry

\begin{abstract}
:
Developments of LiDAR technology are decreasing the unit cost per single point (e.g. single-photo counting). This brings to the possibility of future LiDAR datasets having very dense point clouds. In this work, we process a very dense point cloud ( 200 points per square meter), using three different methods for segmenting single trees and extracting tree positions and other metrics of interest in forestry, such as tree height distribution and canopy area distribution. The three algorithms are tested at decreasing densities, up to a lowest density of $\sim 5$ point per square meter.

Accuracy assessment is done using Kappa, recall, precision and F-Score metrics comparing results with tree positions from groundtruth measurements in six ground plots where tree positions and heights were surveyed manually. Results show that one method provides better Kappa and recall accuracy results for all cases, and that different point densities, in the range used in this study, do not affect accuracy significantly. Processing time is also considered; the method with better accuracy is several times slower than the other two methods and increases exponentially with point density. Best performer gave Kappa $=0.7$. The implications of metrics for determining the accuracy of results of point positions' detection is reported. Motives for the different performances of the three methods is discussed and further research direction is proposed.
\end{abstract}

\section{INTRODUCTION}

\subsection{LiDAR for trees}

In forestry applications LiDAR has a prominent role. It is the only technology that can provide vertical structure information, from the top of the canopy, down to the last feature, i.e. the ground surface. The laser pulse exists in a volumetric space, as it is carried by a beam, which has a characteristic divergence angle, thus a definite footprint if projected on a hypothetical plane perpendicular to the beam's direction. The effective 3D space where the pulse can interact with targets is therefore more like a cone, where the emitter gate can be considered the apex of the cone and the base is the footprint, whose area grows with distance from the emitter.

Tree canopy is a complex structure, which provides multiple targets at various orientations that cause a laser beam to be reflected- e.g. leaves, branches. Gaps in canopy allow part or all of the beam to reach the ground surface. Depending on several factors, the minimum distance for discriminating two targets closely located along the beam's path is between 15 and $30 \mathrm{~cm}$, (Baltsavias, 1999; Mallet and Bretar, 2009). Multiple reflections (echoes) allow for the accurate representation of the vertical tree structure, and of points at ground level that provide an accurate representation of the terrain surface, which is crucial for further processing (Kobal et al., 2015; Lu et al., 2014).
Tree segmentation methods classify LiDAR points to single tree IDs. It is an important part of processing point clouds. Knowing the distribution of trees in forests and in urban areas can be a valid support for providing geospatial information for land management, mapping, and decision making (Piragnolo et al., 2014). Much research focuses on using LiDAR for estimating tree parameters, e.g. volume, vertical distribution, leaf area index etc... These data are a valid help in the estimation of several phenomena linked such parameters, from climate change to urban heat islands, from biodiversity to invasive species, and many others.

Trends in automatic tree detection from LiDAR improved automation in processing LiDAR for forestry applications. Many methods require a canopy height model, which is segmented using watershed and region-growing algorithms. Others use templates and similarity measures to detect best possibilities of tree positions (Pirotti et al., 2016). A multi-scale template matching approach for tree detection and measurement in (Korpela et al., 2007); elliptical and other templates are used in this study to represent tree models. Koch et al., (2006) identified tree positions and canopy crowns using a combination of a pouring algorithm, knowledge-based assumptions on the shape of trees, and a final detection of the crown edges. Many methods are limited in case of dominated trees (trees growing under bigger trees) - this problem has been partly solved by considering morphological indices of tree structure (Barilotti et al., 2007) or full-waveform interpretation (Pirotti et al., 2014; Reitberger et al., 2009). 
Several investigations have been carried out for single tree classification in point clouds. Wavelet analysis in space has been proposed to determine tree position, height and crown diameter of single tree in point clouds (Falkowski et al., 2006). A review of additional recent methods can be found in (Zhen et al., 2016).

In this paper, we report three methods for tree segmentation and localization, and we compare results with the location of trees surveyed on the ground.

\section{MATERIALS AND METHOD}

\subsection{Study area and dataset}

The study area is located in Slovenia, in a forested area with mostly beech trees (Fagus sylvatica) and Norway spruce (Picea abies) see Figure 1 for a geographical overview.

Six circular plots with size of $4000 \mathrm{~m}^{2}$ and radii of $\sim 35.8 \mathrm{~m}$ were sampled for tree locations, species, height and diameter at breast height. For the present work tree location was the main variable of interest, but height distribution of extracted trees was also compared with the one from actual trees. The LiDAR data were acquired in October 2013 with a Riegl LM5600 sensor, capable of recording multiple returns. The point density of the dataset is $\sim 200$ points per square meter, with minor differences per plot (see Table 1)

Table 1. Test plot characteristics

\begin{tabular}{llll}
\hline PlotID & $\begin{array}{l}\text { N. Points } \\
(\mathbf{x 1 0 0 0})\end{array}$ & $\begin{array}{l}\text { Density } \\
\left(\mathbf{P o i n t s}_{\mathbf{m}} \mathbf{m}^{\mathbf{}}\right)\end{array}$ & N. Trees \\
\hline ABIES_50 & 653 & 127 & 72 \\
ABIES_CON & 993 & 193 & 130 \\
FAGUS_50 & 758 & 147 & 83 \\
FAGUS_CON & 1134 & 220 & 129 \\
PICEA_50 & 680 & 132 & 86 \\
PICEA_CON & 957 & 186 & 216 \\
\hline
\end{tabular}

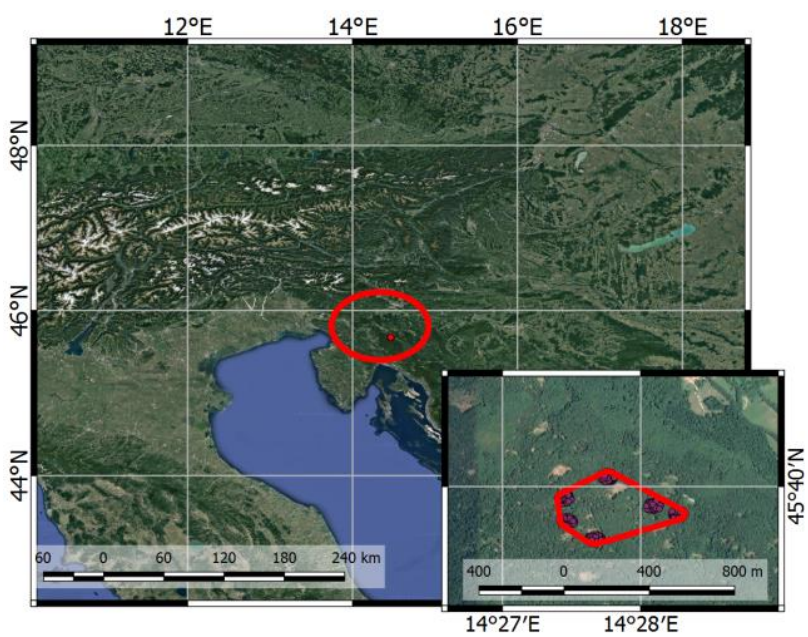

Figure 1. Study area

\subsection{Method}

Three tree segmentation methods were used and are described in detail in the following paragraph - they will be referred to as method "DalPonte", "watershed" and "Li2012" from type and main authors. All methods are implemented in the "lidR" package in R (Roussel and Auty, 2017). The first two methods presented require a normalized digital surface model (nDSM) which in forested areas is referred to as a canopy height model (CHM). For normalization a classification of ground points in the point cloud is a necessary pre-processing step.

2.2.1 Ground points classification: Ground points are necessary to create a digital terrain model (DTM) which is used to normalize the point heights to values relative to the ground. To classify ground points we used an iterative method consisting on an initial grid with a user-defined cell size which is refined up to the required cell size (Pirotti et al., 2013). The initial cell size was set to $5 \mathrm{~m}$ as the point density was never below 1 points per square meter. This size allowed at least one ground point to be in the cell. The final cell size was $1 \mathrm{~m}$. Erosion and dilation removed false positives at each iteration and provided the final DTM at last iteration.

2.2.2 Tree segmentation: Three methods were tested: they are briefly described with references for further reading. The first two methods require a CHM and have one common parameter the need to be set: the minimum canopy height value below which a pixel cannot be considered a crown. This allows to avoid false positives from understorey vegetation. The default value is $2 \mathrm{~m}$, but in our case we changed it to $5 \mathrm{~m}$, as the trees that were surveyed in the 6 plots have a minimum height of $5.63 \mathrm{~m}$. All other parameters were left to their default value. The first method (DalPonte), referenced in Dalponte and Coomes, (2016), consists in using local maxima and a regiongrowing algorithm over the canopy height model (CHM) and the normalized point cloud. The cloud was normalized using a surface created with the ground points detected as described in the previous paragraph. The surface model was a mesh created with Delaunay triangulation. A low-pass filter is then applied to the CHM, with a window of $3 \times 3$ cells to remove peaks and smooth the surface. Initial seed points (equation 1) are then defined using a moving window approach: if the central point to a $5 \times 5$ cells window is the local maximum and if it is higher than a certain threshold, which in this study was set to $5 \mathrm{~m}$ :

$$
S=\left\{s_{1}, s_{2}, \ldots, s_{N}\right\}
$$

where S are seed points. They are then used to define the initial regions. Labels, L, are applied to a map and are defined as:

$$
L_{i, j}=\left\{\begin{array}{c}
k \Rightarrow S_{i, j} \\
0
\end{array}\right.
$$

where $k$ is the initially labelled points and the region growing algorithm proceeds by considering the cells with no label $\left(\mathrm{L}_{\mathrm{i}, \mathrm{j}}=\right.$ 0 ) immediately neighbouring the $k$ labelled cells and assigning the label of the neighbour only if it meets certain criteria related to distance and height of CHM. From each region a threshold selection method (Otsu, 1979) is applied to only first return points. A convex hull is applied to each set of points with a unique label, thus defining single tree crowns - all points area then assigned a tree ID corresponding to the polygon that they overlap.

The second method (watershed) is based on the watershed algorithm, which uses the canopy surface model to segment regions according to an inverted CHM. The local maxima are used as seed points in this method as well. The size of each watershed is kept between a minimum and maximum value to avoid false canopies to be defined.

The third method (Li2012) does not require a CHM and uses purely a graph (Li et al., 2012). No minimum canopy height 
value is defined like the other two methods, and we realised that some low vegetation is defined as tree. Therefore we set a postprocessing step to remove low vegetation with the same height threshold of $5 \mathrm{~m}$.

2.2.3 Extraction of forest parameters: The product of tree segmentation is to have all points in the point cloud labelled according to the tree that they belong to, or a null label if they do not belong to any tree. The label corresponds to a unique tree ID. The position of each tree from the point cloud $\left(\mathrm{P}_{\mathrm{pc}}\right)$ is extracted by taking, for each set of points with a unique tree ID, the point with highest $\mathrm{Z}$ value. Its coordinates are considered the position of the tree apex for that tree. For each plot we also extract the frequency distribution for the tree heights and for the canopy areas to compare it to measured values.
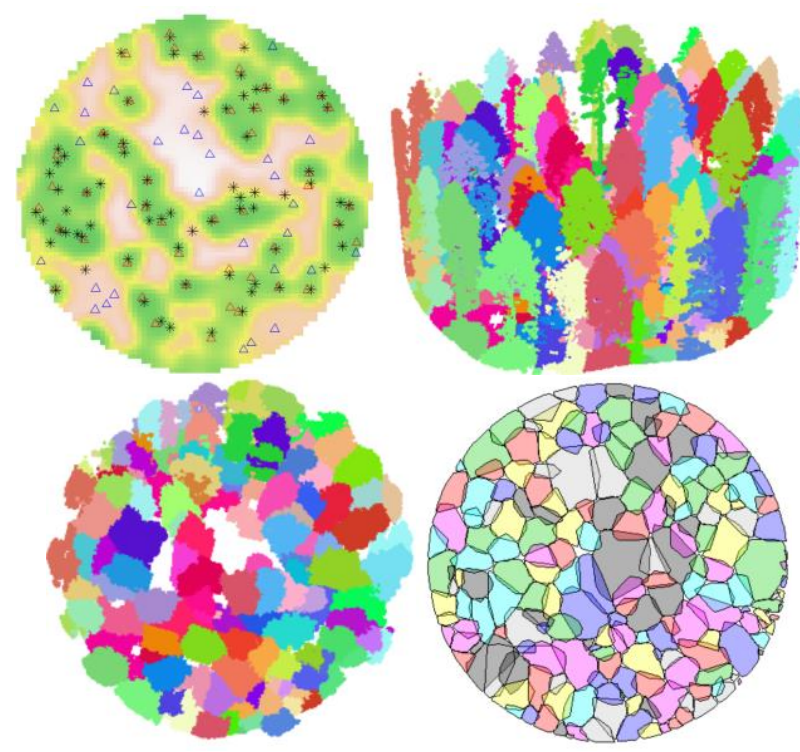

Figure 2. Four views of single plot with segmented points assigned to unique tree IDs, their positions (top left: red triangles are true positives, blue triangles false positives) and their canopies.

2.2.4 Accuracy assessment: Assessing the accuracy of calculated tree locations is a fundamental aspect and not an easy one. Eysn et al., 2015, and Pirotti, 2010 proposed methods that define commission and omission errors by matching calculated tree positions with ground-measured tree positions depending on a minimum distance. Pirotti, (2010) uses a distance threshold that is derived from the average tree distance of real trees $(2.3 \mathrm{~m}$ in the reported research). The method by Eysn et al. (2015) uses a more complex decision procedure that weights distance and tree height differences.

To assess if the detected trees correspond with existing trees, we compared them with trees from the sampling campaign. The position of the real trees $\left(\mathrm{P}_{\text {real }}\right)$ is known, and we match them with tree positions extracted from the point cloud $\left(\mathrm{P}_{\mathrm{pc}}\right)$. Matching is done by finding its closest neighbour from the set of $P_{\text {real. }}$ It is basically an intersection procedure of two sets, and tree position $x$ belongs to one of the following classes:

$$
\begin{gathered}
T P=\left\{x \in P_{\text {real }} \wedge x \in P_{\text {seg }}\right\} \\
T N=\left\{x \notin P_{\text {real }} \mid x \notin P_{\text {seg }}\right\} \\
F P=\left\{P_{\text {seg }} \in P_{\text {real }} \mid x \notin P_{\text {seg }}\right\} \\
F N=\left\{x \notin P_{\text {real }} \mid x \in P_{\text {seg }}\right\}
\end{gathered}
$$

where $\mathrm{P}_{\text {real }}$ is the surveyed point set and $\mathrm{P}_{\mathrm{pc}}$ is the lidar-inferred tree location, FP is false positives, or commission errors, $\mathrm{FN}$ is false negatives, or omission errors, TP is true positives, i.e. the trees from segmentation matching the real trees in terms of location. Matching was done by calculating the distance between each $\mathrm{P}_{\mathrm{pc}}$ the nearest $\mathrm{P}_{\text {real }}$; TP are the points which meet the following two criteria:

a) distance to nearest real tree is below a radius of their height divided by 10 , on other terms, we accounted for a larger threshold for taller trees. A tree which is $10 \mathrm{~m}$ tall will be matched successfully if a tree is found at a distance equal to or less than one meter. A $30 \mathrm{~m}$ tall tree can be matched at a distance to a real tree of $3 \mathrm{~m}$.

b) The difference in height of the matched trees is not above $10 \%$ - e.g. a $30 \mathrm{~m}$ tall tree cannot be considered matched to a tree whose ground-measured height is below $27 \mathrm{~m}$ or above 33 $\mathrm{m}$.

In equation 3, elements belong to both sets if they meet the two criteria above. The thresholds are necessary as ground sampling identifies the tree at the bottom of the trunk, whereas LiDAR methods identify the location at the apex of the crown, and these two might naturally be different. Another critical aspect is that any manual survey is prone to errors, which are not easily quantified, as they depend on numerous factors, the subjective training of personnel also playing a role. Nevertheless, for these initial tests we ignored these factors. A last component of accuracy is the True Negatives (TN) - these are calculated by subtracting the number of TP, FP and FN from the number of pixels in the CHM. This leads to "unnatural" high values of Kappa index of accuracy, but we are interested in the relative accuracy change between trial, and the TN component is necessary to calculate the index.

From TP, FP and FN values we calculate the precision (Pr), recall $(\mathrm{Re}), \mathrm{F}-\mathrm{Score}$ and Kappa index metrics:

$$
\begin{gathered}
\operatorname{Pr}=\frac{T P}{T P+F P} \\
\operatorname{Re}=\frac{T P}{T P+F N} \\
F-\text { index }=2 \cdot \frac{\operatorname{Pr} \cdot \operatorname{Re}}{\operatorname{Pr}+\operatorname{Re}} \\
K=\frac{P_{o}-P_{e}}{1-P_{e}}
\end{gathered}
$$

Where Po is $\mathrm{TP}+\mathrm{TN}$ and $\mathrm{Pe}$ is expected matches from chance, see (Pontius and Millones, 2011). We chose these indices adding from (Pirotti, 2010) due to the unbalance that can be created by calculating only Kappa and because we are interested in observing the behaviour of these accuracy metrics to assess also which reflects better the goodness of results.

2.2.5 Point density reduction: A note on how point density was reduced is important. A simple reduction resulting from keeping $n$ points every $N$ points is not the best approach for simulating lower pulse repetition rates as it does not account for the multiple echoes which vary in number for each pulse. 
Therefore our approach was to apply keep $n$ pulses every $N$, and therefore keep or remove all the relative points resulting from the echoes. This approach resembles more closely different pulse rates in a LiDAR flight, or higher relative flying heights.

\section{RESULTS AND DISCUSSION}

3.1.1 Overview of accuracy metrics: The results in terms of accuracy indices, precision (Pr), recall (Re), F-Score and Kappa are shown in Figure 3, where all results are plotted against point densities for each plot. The F-Score aggregates Pr and Re values and it does not show any particular correlation with density values. This might seem to indicate that point density is not an issue in this particular study case, but several critical points have to be discussed. First of all the procedure for defining the accuracy, described in the last part of the methods section, suffers from several drawbacks. One might think that more detected trees, even if most are false positives, might increase the overall measure for accuracy (F-Score). This can be tested checking correlation between the number of trees detected and the F-Score, aggregating by plot and by method. Table 2 below shows the results.

There are some cases where there is very high correlation. This correlation indicates that the accuracy measure is somehow dependent from the number of detected trees (both correct true positives and errors, i.e. false positives). This is something that must be corrected, as an accuracy measure should be independent from the number of features in the population that is being assessed. This means that F-Score is not the best metric to test this type of results. For this reason we used the Kappa index of accuracy, were random true positives are taken into account by weighting with expected prediction by chance $(\mathrm{Pe})-$ equation 4. The results show that there is not a significant difference in results of Kappa when considering the point density range from $\sim 5$ to $\sim 200$. To see effective differences the point density should probably be lowered to $<5$ point per square meter, and this will be the topic of future research. When considering the methods, Li 2012 performs better in all plots. The drawback of this method, as discussed further later, is the processing time. An analysis of Type I errors, false positives, which are identified with index of precision (Pr), shows a less marked separation between methods, as is seen from larger envelopes. Li 2012 has lower Pr compared to the other two methods for all plots.

Table 2. COR=correlation (Pearson) values of F-Score and number of detected trees; ordered by COR values.

\begin{tabular}{lll}
\hline PlotID & Method & COR \\
FAGUS_CON & dalponte2016 & 0.00 \\
PICEA_50 & Li2012 & 0.01 \\
PICEA_50 & dalponte2016 & 0.06 \\
ABIES_50 & Li2012 & 0.08 \\
ABIES_CON & Li2012 & 0.24 \\
FAGUS_50 & Li2012 & 0.29 \\
PICEA_50 & watershed & 0.61 \\
ABIES_CON & dalponte2016 & 0.66 \\
FAGUS_CON & watershed & 0.73 \\
ABIES_CON & watershed & 0.76 \\
FAGUS_CON & Li2012 & 0.78 \\
ABIES_50 & watershed & 0.79 \\
PICEA_CON & Li2012 & 0.81 \\
FAGUS_50 & dalponte2016 & 0.82 \\
PICEA_CON & dalponte2016 & 0.84 \\
ABIES_50 & dalponte2016 & 0.85 \\
PICEA_CON & watershed & 0.87 \\
\hline
\end{tabular}

Type II errors, false negatives, i.e. the real tree positions which are missed - not detected - is analysed by the recall (R) index. In this case the Li 2012 performs best. For all metrics, the method by Dalponte and Coomes, (2016) performs second best, and best between the two methods which require $\mathrm{CHM}$ for initial seed points.

3.1.2 Processing time: An analysis of processing time is reported, because such an information weights on decisions on what method to adopt for processing data. The last row of Figure 3 show that the two methods using CHM, Watershed and DalPonte, are several times much faster than Li2012. A detail is shown below in Figure 4 of the two "quick" methods. They follow a linear relationship with density. The "slow" method, Li2012, grows exponentially with point density. Each ground plot has a different curve. Therefore it can be inferred that processing time for this method is correlated to both the total number of point and to the density. In other words the time for completing a process depends not only on the total number of points, but also on density.

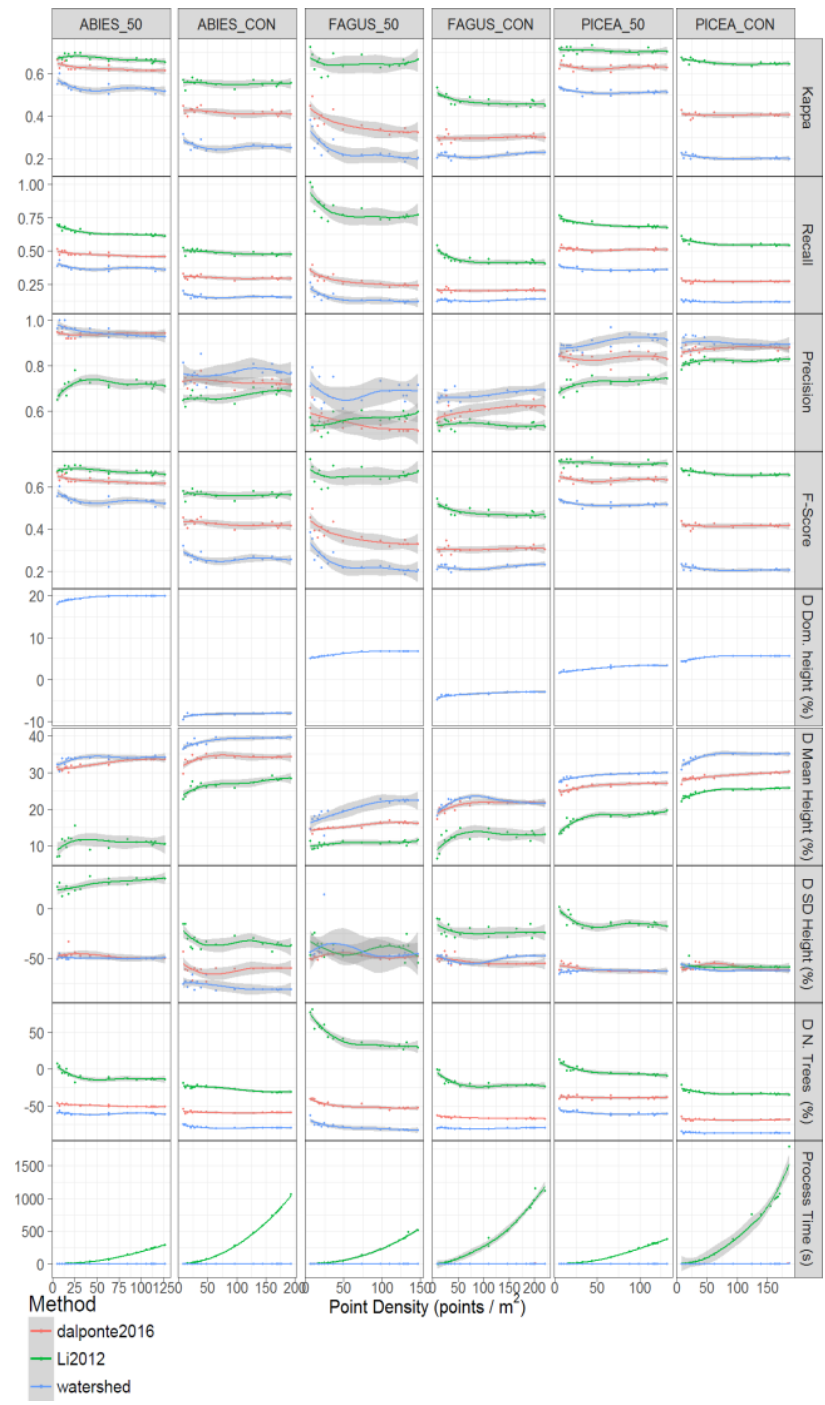

Figure 3. Accuracy metrics and processing time at different point densities and for different plots - more details in table 3 - processing time is in seconds. 
This method also has a parameter called $\mathrm{R}$, which influences the processing time heavily. It is the maximum radius to consider for a tree canopy. Its default value is 10 , which is reasonable in this study and in most cases in mature forests; we therefore left this value. Lower $\mathrm{R}$ values will improve processing time, but must be changed with care depending on the characteristics of the forest. A point density of $\sim 200$ points per square meter would mean to expect $\sim 62800$ points per tree, and the method will have to assign to all points iteratively the tree ID. This study came to an interesting and very practical conclusion that very high point density is not necessary for best results. The other two methods are much faster because they base the first point selection on the CHM. Processing time for these two methods is directly proportional to the number of points.

3.1.3 Comparison with literature: A comparison of the results from the literature of the Li2012 method with the results from this study are also reported. A comparable point density was used; Li et al., (2012) used a dataset with $>6$ points $/ \mathrm{m}^{2}$ and the results gave recall $=0.86$, precision $=0.94$ and F-Score $=0.9$. In this study, using a comparable point density $\left(7\right.$ points $\left./ \mathrm{m}^{2}\right)$, gives recall $=0.68$, precision $=0.72$ and F-Score $=0.7$. The dataset in this study is quite different, with larger plots and more complex vegetation structure. This might lead to more difficult detection of dominated trees, which are notably the hardest features to detect and segment.

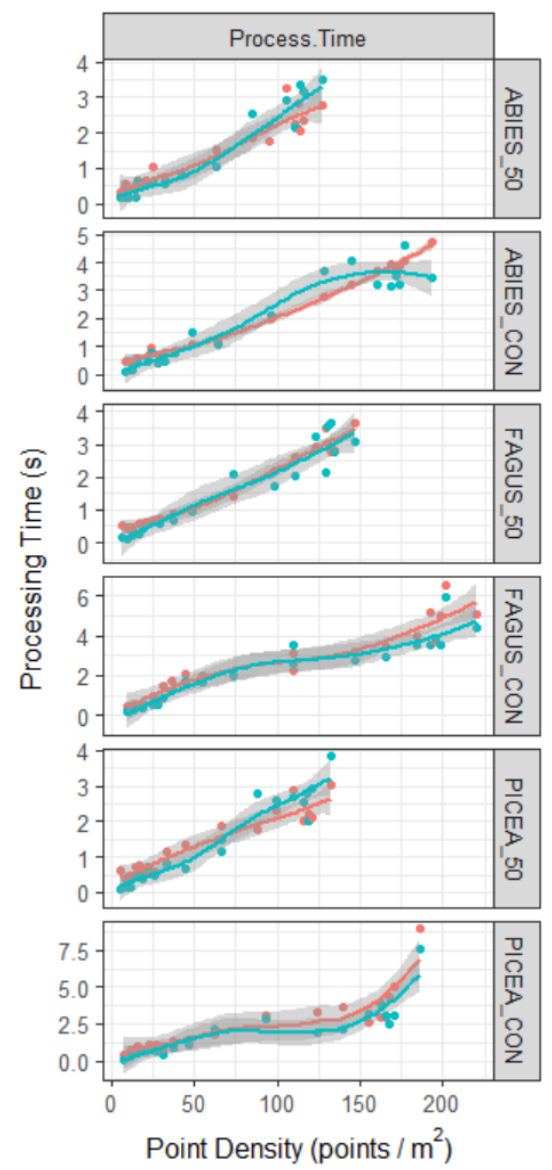

Figure 4. Details of processing time for the two quickest methods, DalPonte (red) and watershed (blue) legend is same as figure 3 .
Table 3. Definition of some metrics in plot rows in Figure 3.

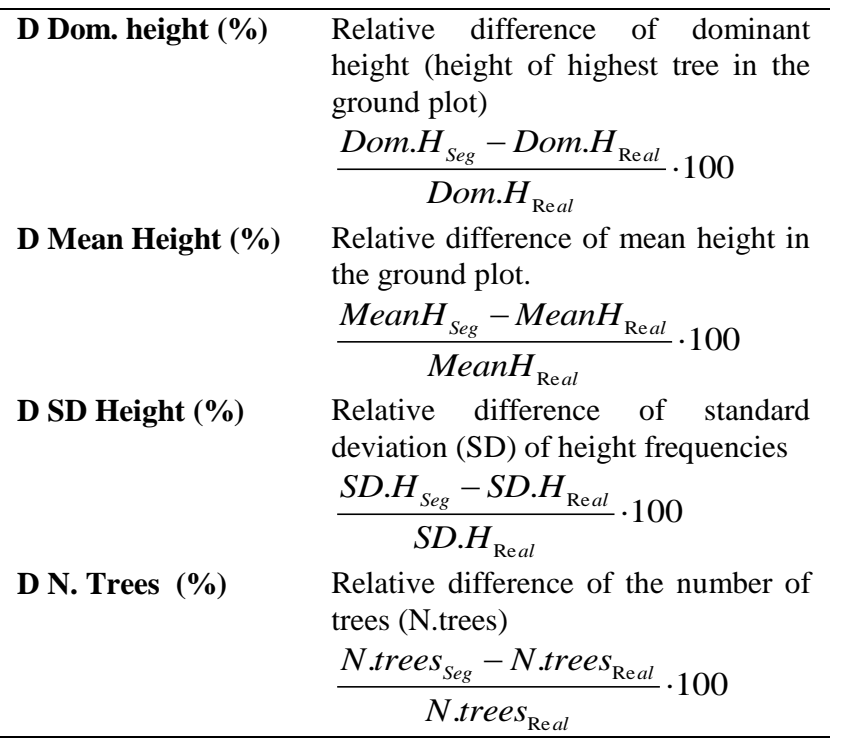

\section{CONCLUSIONS}

In this paper we report results on a comparison of three methods for locating trees and determine also the height distribution in a forest area. The results were assessed by considering correct matches the detected trees that fell within a distance and had similar height with a tree from tree locations sampled on the ground. Accuracy metrics show The results show that there is not a significant difference in results of Precision, Recall and Kappa when considering the point density range from $\sim 5$ to 200. To see effective differences the point density should probably be lowered to $<5$ point per square meter, and this will be the topic of future research. One of the three methods gave significant better results then the other two, even if a lower accuracy with respect to the original literature of the method. The other two methods are much faster than this method, but give worse results, in terms of detecting tree positions.

Further investigations will review the differences between methods in terms of point densities lower than 5 points per square meter, and will also determine how the estimation of forest parameter can be carried out with the best combination of method and point density.

\section{REFERENCES}

Baltsavias, E.P., 1999. Airborne laser scanning: Basic relations and formulas. ISPRS Journal of Photogrammetry and Remote Sensing, 54, 199-214. doi:10.1016/S0924-2716(99)00015-5

Barilotti, A., Sepic, F., Abramo, E., Crosilla, F., 2007. Improving the morphological analysis for tree extraction: a dynamic approach to lidar data. International Archives of the Photogrammetry, Remote Sensing and Spatial Information Sciences, XXXVI/3-W5, 33-36.

Dalponte, M., Coomes, D.A., 2016. Tree-centric mapping of forest carbon density from airborne laser scanning and hyperspectral data. Methods in Ecology and 


\section{Evolution, 7, 1236-1245. doi:10.1111/2041-210X.12575}

Eysn, L., Hollaus, M., Lindberg, E., Berger, F., Monnet, J.-M., Dalponte, M., Kobal, M., Pellegrini, M., Lingua, E., Mongus, D., Pfeifer, N., 2015. A Benchmark of Lidar-Based Single Tree Detection Methods Using Heterogeneous Forest Data from the Alpine Space. Forests, 6, 1721-1747. doi:10.3390/f6051721

Falkowski, M.J., Smith, A.M.S., Hudak, A.T., Gessler, P.E., Vierling, L.A., Crookston, N.L., 2006. Automated estimation of individual conifer tree height and crown diameter via two-dimensional spatial wavelet analysis of lidar data. Canadian Journal of Remote Sensing, 32, 153-161.

Kobal, M., Bertoncelj, I., Pirotti, F., Dakskobler, I., Kutnar, L., 2015. Using Lidar Data to Analyse Sinkhole Characteristics Relevant for Understory Vegetation under Forest Cover - Case Study of a High Karst Area in the Dinaric Mountains. PLOS ONE, 10, e0122070. doi:10.1371/journal.pone.0122070

Koch, B., Heyder, U., Weinacker, H., 2006. Detection of individual tree crowns in airborne lidar data. Photogrammetric Engineering \& Remote Sensing, 72, 357-363.

Korpela, I., Dahlin, B., Schäfer, H., Bruun, E., Haapaniemi, F., Honkasalo, J., Ilvesniemi, S., Kuutti, V., Linkosalmi, M., Mustonen, J., Salo, M., Suomi, O., Virtanen, H., 2007. Single-tree forest inventory using lidar and aerial images for 3D treetop positioning, species recognition, height and crown width estimation. International Archives of the Photogrammetry, Remote Sensing and Spatial Information Sciences, 36, 227-233.

Li, W., Guo, Q., Jakubowski, M.K., Kelly, M., 2012. A New Method for Segmenting Individual Trees from the Lidar Point Cloud. Photogrammetric Engineering \& Remote Sensing, 78, 75-84. doi:10.14358/PERS.78.1.75

Lu, X., Guo, Q., Li, W., Flanagan, J., 2014. A bottom-up approach to segment individual deciduous trees using leaf-off lidar point cloud data. ISPRS Journal of Photogrammetry and Remote Sensing, 94, 1-12. doi:10.1016/j.isprsjprs.2014.03.014

Mallet, C., Bretar, F., 2009. Full-waveform topographic lidar: State-of-the-art. ISPRS Journal of Photogrammetry and Remote Sensing, 64, 1-16. doi:10.1016/j.isprsjprs.2008.09.007

Otsu, N., 1979. A threshold selection method from graylevel histograms. IEEE Transactions on Systems, Man, and Cybernetics, 9, 62-66. doi:10.1109/TSMC.1979.4310076

Piragnolo, M., Pirotti, F., Guarnieri, A., Vettore, A., Salogni, G., 2014. Geo-Spatial Support for Assessment of Anthropic Impact on Biodiversity. ISPRS International Journal of Geo-Information, 3, 599-618. doi:10.3390/ijgi3020599

Pirotti, F., 2010. Assessing a Template Matching Approach for Tree Height and Position Extraction from Lidar-Derived Canopy Height Models of Pinus Pinaster

\section{Stands. Forests, 1, 194-208. doi:10.3390/f1040194}

Pirotti, F., Guarnieri, A., Vettore, A., 2013. Vegetation filtering of waveform terrestrial laser scanner data for DTM production. Applied Geomatics, 5, 311-322. doi:10.1007/s12518-013-0119-3

Pirotti, F., Laurin, G., Vettore, A., Masiero, A., Valentini, R., 2014. Small Footprint Full-Waveform Metrics Contribution to the Prediction of Biomass in Tropical Forests. Remote Sensing, 6, 9576-9599. doi:10.3390/rs6109576

Pirotti, F., Travaglini, D., Giannetti, F., Kutchartt, E., Bottalico, F., Chirici, G., 2016. Kernel Feature CrossCorrelation for Unsupervised Quantification of Damage from Windthrow in Forests. ISPRS - International Archives of the Photogrammetry, Remote Sensing and Spatial Information Sciences,. doi:10.5194/isprsarchives-XLI-B7-17-2016

Pontius, R.G., Millones, M., 2011. Death to Kappa: birth of quantity disagreement and allocation disagreement for accuracy assessment. International Journal of Remote Sensing, 32, 4407-4429. doi:10.1080/01431161.2011.552923

Reitberger, J., Schnörr, C., Krzystek, P., Stilla, U., 2009. 3D segmentation of single trees exploiting full waveform LIDAR data. ISPRS Journal of Photogrammetry and Remote Sensing, 64, 561-574. doi:10.1016/j.isprsjprs.2009.04.002

Roussel, J.-R., Auty, D., 2017. lidR: Airborne LiDAR Data Manipulation and Visualization for Forestry Applications.

Zhen, Z., Quackenbush, L., Zhang, L., 2016. Trends in Automatic Individual Tree Crown Detection and Delineation-Evolution of LiDAR Data. Remote Sensing, 8, 333. doi:10.3390/rs8040333 